\title{
Martingale inequalities for spline sequences
}

\section{Markus Passenbrunner ${ }^{1}[$}

Received: 6 February 2019 / Accepted: 21 March 2019 / Published online: 30 March 2019

(c) The Author(s) 2019

\section{Abstract}

We show that D. Lépingle's $L_{1}\left(\ell_{2}\right)$-inequality

$$
\left\|\left(\sum_{n} \mathbb{E}\left[f_{n} \mid \mathscr{F}_{n-1}\right]^{2}\right)^{1 / 2}\right\|_{1} \leq 2 \cdot\left\|\left(\sum_{n} f_{n}^{2}\right)^{1 / 2}\right\|_{1}, \quad f_{n} \in \mathscr{F}_{n},
$$

extends to the case where we substitute the conditional expectation operators with orthogonal projection operators onto spline spaces and where we can allow that $f_{n}$ is contained in a suitable spline space $\mathscr{S}\left(\mathscr{F}_{n}\right)$. This is done provided the filtration $\left(\mathscr{F}_{n}\right)$ satisfies a certain regularity condition depending on the degree of smoothness of the functions contained in $\mathscr{S}\left(\mathscr{F}_{n}\right)$. As a by-product, we also obtain a spline version of $H_{1}$-BMO duality under this assumption.

Keywords Martingale inequalities · Polynomial spline spaces · Orthogonal projection operators

Mathematics Subject Classification 65D07 · 60G42 · 42C10

\section{Introduction}

This article is part of a series of papers that extend martingale results to polynomial spline sequences of arbitrary order (see e.g. [11,14,16-19,22]). In order to explain those martingale type results, we have to introduce a little bit of terminology: Let $k$ be a positive integer, $\left(\mathscr{F}_{n}\right)$ an increasing sequence of $\sigma$-algebras of sets in $[0,1]$ where each $\mathscr{F}_{n}$ is generated by a finite partition of $[0,1]$ into intervals of positive length. Moreover, define the spline space

$$
\mathscr{S}_{k}\left(\mathscr{F}_{n}\right)=\left\{f \in C^{k-2}[0,1]: f \text { is a polynomial of order } k \text { on each atom of } \mathscr{F}_{n}\right\}
$$

Markus Passenbrunner

markus.passenbrunner@jku.at

1 Institute of Analysis, Johannes Kepler University Linz, Altenberger Strasse 69, 4040 Linz, Austria 
and let $P_{n}^{(k)}$ be the orthogonal projection operator onto $\mathscr{S}_{k}\left(\mathscr{F}_{n}\right)$ with respect to the $L_{2}$ inner product on $[0,1]$ with the Lebesgue measure $|\cdot|$. The space $\mathscr{S}_{1}\left(\mathscr{F}_{n}\right)$ consists of piecewise constant functions and $P_{n}^{(1)}$ is the conditional expectation operator with respect to the $\sigma$-algebra $\mathscr{F}_{n}$. Similarly to the definition of martingales, we introduce the following notion: let $\left(f_{n}\right)_{n \geq 0}$ be a sequence of integrable functions. We call this sequence a $k$-martingale spline sequence (adapted to $\left(\mathscr{F}_{n}\right)$ ) if, for all $n$,

$$
P_{n}^{(k)} f_{n+1}=f_{n}
$$

For basic facts about martingales and conditional expectations, we refer to [15].

Classical martingale theorems such as Doob's inequality or the martingale convergence theorem in fact carry over to $k$-martingale spline sequences corresponding to arbitrary filtrations $\left(\mathscr{F}_{n}\right)$ of the above type, just by replacing conditional expectation operators by the projection operators $P_{n}^{(k)}$. Indeed, we have

(i) (Shadrin's theorem) there exists a constant $C_{k}$ depending only on $k$ such that

$$
\sup _{n}\left\|P_{n}^{(k)}: L_{1} \rightarrow L_{1}\right\| \leq C_{k}
$$

(ii) (Doob's weak type inequality for splines)

there exists a constant $C_{k}$ depending only on $k$ such that for any $k$-martingale spline sequence $\left(f_{n}\right)$ and any $\lambda>0$,

$$
\left|\left\{\sup _{n}\left|f_{n}\right|>\lambda\right\}\right| \leq C_{k} \frac{\sup _{n}\left\|f_{n}\right\|_{1}}{\lambda},
$$

(iii) (Doob's $L_{p}$ inequality for splines)

for all $p \in(1, \infty]$ there exists a constant $C_{p, k}$ depending only on $p$ and $k$ such that for all $k$-martingale spline sequences $\left(f_{n}\right)$,

$$
\left\|\sup _{n}\left|f_{n}\right|\right\|_{p} \leq C_{p, k} \sup _{n}\left\|f_{n}\right\|_{p}
$$

(iv) (Spline convergence theorem)

if $\left(f_{n}\right)$ is an $L_{1}$-bounded $k$-martingale spline sequence, then $\left(f_{n}\right)$ converges almost surely to some $L_{1}$-function,

(v) (Spline convergence theorem, $L_{p}$-version)

for $1<p<\infty$, if $\left(f_{n}\right)$ is an $L_{p}$-bounded $k$-martingale spline sequence, then $\left(f_{n}\right)$ converges almost surely and in $L_{p}$.

Property (i) was proved by Shadrin in the groundbreaking paper [22]. We also refer to the paper [25] by von Golitschek, who gives a substantially shorter proof of (i). Properties (ii) and (iii) are proved in [19] and properties (iv) and (v) in [14], but see also [18], where it is shown that, in analogy to the martingale case, the validity of (iv) and (v) for all $k$-martingale spline sequences with values in a Banach space $X$ characterize the Radon-Nikodým property of $X$ (for background information on that material, we refer to the monographs $[6,20])$. 
Here, we continue this line of transferring martingale results to $k$-martingale spline sequences and extend Lépingle's $L_{1}\left(\ell_{2}\right)$-inequality [12], which reads

$$
\left\|\left(\sum_{n} \mathbb{E}\left[f_{n} \mid \mathscr{F}_{n-1}\right]^{2}\right)^{1 / 2}\right\|_{1} \leq 2 \cdot\left\|\left(\sum_{n} f_{n}^{2}\right)^{1 / 2}\right\|_{1}
$$

provided the sequence of (real-valued) random variables $f_{n}$ is adapted to the filtration $\left(\mathscr{F}_{n}\right)$, i.e. each $f_{n}$ is $\mathscr{F}_{n}$-measurable. Different proofs of (1.1) were given by Bourgain [3, Proposition 5], Delbaen and Schachermayer [4, Lemma 1] and Müller [13, Proposition 4.1]. The spline version of inequality (1.1) is contained in Theorem 4.1.

This inequality is an $L_{1}$ extension of the following result for $1<p<\infty$, proved by Stein [24], that holds for arbitrary integrable functions $f_{n}$ :

$$
\left\|\left(\sum_{n} \mathbb{E}\left[f_{n} \mid \mathscr{F}_{n-1}\right]^{2}\right)^{1 / 2}\right\|_{p} \leq a_{p}\left\|\left(\sum_{n} f_{n}^{2}\right)^{1 / 2}\right\|_{p}
$$

for some constant $a_{p}$ depending only on $p$. This can be seen as a dual version of Doob's inequality $\left\|\sup _{\ell}\left|\mathbb{E}\left[f \mid \mathscr{F}_{\ell}\right]\right|\right\|_{p} \leq c_{p}\|f\|_{p}$ for $p>1$, see [1]. Once we know Doob's inequality for spline projections, which is point (iii) above, the same proof as in [1] works for spline projections if we use suitable positive operators $T_{n}$ instead of $P_{n}^{(k)}$ that also satisfy Doob's inequality and dominate the operators $P_{n}^{(k)}$ pointwise (cf. Sects. 3.1, 3.2).

The usage of those operators $T_{n}$ is also necessary in the extension of inequality (1.1) to splines. Lépingle's proof of (1.1) rests on an idea by Herz [10] of splitting $\mathbb{E}\left[f_{n} \cdot h_{n}\right]$ (for $f_{n}$ being $\mathscr{F}_{n}$-measurable) by Cauchy-Schwarz after introducing the square function $S_{n}^{2}=\sum_{\ell \leq n} f_{\ell}^{2}$ :

$$
\left(\mathbb{E}\left[f_{n} \cdot h_{n}\right]\right)^{2} \leq \mathbb{E}\left[f_{n}^{2} / S_{n}\right] \cdot \mathbb{E}\left[S_{n} h_{n}^{2}\right]
$$

and estimating both factors on the right hand side separately. A key point in estimating the second factor is that $S_{n}$ is $\mathscr{F}_{n}$-measurable, and therefore, $\mathbb{E}\left[S_{n} \mid \mathscr{F}_{n}\right]=S_{n}$. If we want to allow $f_{n} \in \mathscr{S}_{k}\left(\mathscr{F}_{n}\right), S_{n}$ will not be contained in $\mathscr{S}_{k}\left(\mathscr{F}_{n}\right)$ in general. Under certain conditions on the filtration $\left(\mathscr{F}_{n}\right)$, we will show in this article how to substitute $S_{n}$ in estimate (1.3) by a function $g_{n} \in \mathscr{S}_{k}\left(\mathscr{F}_{n}\right)$ that enjoys similar properties to $S_{n}$ and allows us to proceed (cf. Sect. 3.4, in particular Proposition 3.4 and Theorem 3.6). As a by-product, we obtain a spline version (Theorem 4.2) of C. Fefferman's theorem [7] on $H^{1}$-BMO duality. For its martingale version, we refer to A. M. Garsia's book [8] on Martingale Inequalities.

\section{Preliminaries}

In this section, we collect all tools that are needed subsequently. 


\subsection{Properties of polynomials}

We will need Remez' inequality for polynomials:

Theorem 2.1 Let $V \subset \mathbb{R}$ be a compact interval in $\mathbb{R}$ and $E \subset V$ a measurable subset. Then, for all polynomials p of order $k$ (i.e. degree $k-1$ ) on $V$,

$$
\|p\|_{L_{\infty}(V)} \leq\left(4 \frac{|V|}{|E|}\right)^{k-1}\|p\|_{L_{\infty}(E)}
$$

Applying this theorem with the set $E=\left\{x \in V:|p(x)| \leq 8^{-k+1}\|p\|_{L_{\infty}(V)}\right\}$ immediately yields the following corollary:

Corollary 2.2 Let $p$ be a polynomial of order $k$ on a compact interval $V \subset \mathbb{R}$. Then

$$
\left|\left\{x \in V:|p(x)| \geq 8^{-k+1}\|p\|_{L_{\infty}(V)}\right\}\right| \geq|V| / 2 .
$$

\subsection{Properties of spline functions}

For an interval $\sigma$-algebra $\mathscr{F}$ (i.e. $\mathscr{F}$ is generated by a finite collection of intervals having positive length), the space $\mathscr{S}_{k}(\mathscr{F})$ is spanned by a very special local basis $\left(N_{i}\right)$, the so called B-spline basis. It has the properties that each $N_{i}$ is non-negative and each support of $N_{i}$ consists of at most $k$ neighboring atoms of $\mathscr{F}$. Moreover, $\left(N_{i}\right)$ is a partition of unity, i.e. for all $x \in[0,1]$, there exist at most $k$ functions $N_{i}$ so that $N_{i}(x) \neq 0$ and $\sum_{i} N_{i}(x)=1$. In the following, we denote by $E_{i}$ the support of the B-spline function $N_{i}$. The usual ordering of the B-splines $\left(N_{i}\right)$-which we also employ here-is such that for all $i$, inf $E_{i} \leq \inf E_{i+1}$ and $\sup E_{i} \leq \sup E_{i+1}$.

We write $A(t) \lesssim B(t)$ to denote the existence of a constant $C$ such that for all $t$, $A(t) \leq C B(t)$, where $t$ denote all implicit and explicit dependencies the expression $A$ and $B$ might have. If the constant $C$ additionally depends on some parameter, we will indicate this in the text. Similarly, the symbols $\gtrsim$ and $\simeq$ are used.

Another important property of B-splines is the following relation between B-spline coefficients and the $L_{p}$-norm of the corresponding B-spline expansions.

Theorem 2.3 (B-spline stability, local and global) Let $1 \leq p \leq \infty$ and $g=\sum_{j} a_{j} N_{j}$. Then, for all $j$,

$$
\left|a_{j}\right| \lesssim\left|J_{j}\right|^{-1 / p}\|g\|_{L_{p}\left(J_{j}\right)}
$$

where $J_{j}$ is an atom of $\mathscr{F}$ contained in $E_{j}$ having maximal length. Additionally,

$$
\|g\|_{p} \simeq\left\|\left(a_{j}\left|E_{j}\right|^{1 / p}\right)\right\|_{\ell_{p}}
$$

where in both (2.1) and (2.2), the implied constants depend only on the spline order $k$. 
Observe that (2.1) implies for $g \in \mathscr{S}_{k}(\mathscr{F})$ and any measurable set $A \subset[0,1]$

$$
\|g\|_{L_{\infty}(A)} \lesssim \max _{j:\left|E_{j} \cap A\right|>0}\|g\|_{L_{\infty}\left(J_{j}\right)}
$$

We will also need the following relation between the B-spline expansion of a function and its expansion using B-splines of a finer grid.

Theorem 2.4 Let $\mathscr{G} \subset \mathscr{F}$ be two interval $\sigma$-algebras and denote by $\left(N_{\mathscr{G}, i}\right)_{i}$ the $B$ spline basis of the coarser space $\mathscr{S}_{k}(\mathscr{G})$ and by $\left(N_{\mathscr{F}, i}\right)_{i}$ the $B$-spline basis of the finer space $\mathscr{S}_{k}(\mathscr{F})$. Then, given $f=\sum_{j} a_{j} N_{\mathscr{G}, j}$, we can expand $f$ in the basis $\left(N_{\mathscr{F}, i}\right)_{i}$

$$
\sum_{j} a_{j} N_{\mathscr{G}, j}=\sum_{i} b_{i} N_{\mathscr{F}, i},
$$

where for each $i, b_{i}$ is a convex combination of the coefficients $a_{j}$ with $\operatorname{supp} N_{\mathscr{G}}, j \supseteq$ $\operatorname{supp} N_{\mathscr{F}, i}$.

For those results and more information on spline functions, in particular B-splines, we refer to [21] or [5].

\subsection{Spline orthoprojectors}

We now use the B-spline basis of $\mathscr{S}_{k}(\mathscr{F})$ and expand the orthogonal projection operator $P$ onto $\mathscr{S}_{k}(\mathscr{F})$ in the form

$$
P f=\sum_{i, j} a_{i j}\left(\int_{0}^{1} f(x) N_{i}(x) \mathrm{d} x\right) \cdot N_{j}
$$

for some coefficients $\left(a_{i j}\right)$. Denoting by $E_{i j}$ the smallest interval containing both supports $E_{i}$ and $E_{j}$ of the B-spline functions $N_{i}$ and $N_{j}$ respectively, we have the following estimate for $a_{i j}$ [19]: there exist constants $C$ and $0<q<1$ depending only on $k$ so that for each interval $\sigma$-algebra $\mathscr{F}$ and each $i, j$,

$$
\left|a_{i j}\right| \leq C \frac{q^{|i-j|}}{\left|E_{i j}\right|} .
$$

\subsection{Spline square functions}

Let $\left(\mathscr{F}_{n}\right)$ be a sequence of increasing interval $\sigma$-algebras in $[0,1]$ and we assume that each $\mathscr{F}_{n+1}$ is generated from $\mathscr{F}_{n}$ by the subdivision of exactly one atom of $\mathscr{F}_{n}$ into two atoms of $\mathscr{F}_{n+1}$. Let $P_{n}$ be the orthogonal projection operator onto $\mathscr{S}_{k}\left(\mathscr{F}_{n}\right)$. We denote $\Delta_{n} f=P_{n} f-P_{n-1} f$ and define the spline square function

$$
S f=\left(\sum_{n}\left|\Delta_{n} f\right|^{2}\right)^{1 / 2} .
$$


We have Burkholder's inequality for the spline square function, i.e. for all $1<p<\infty$ [16], the $L_{p}$-norm of the square function $S f$ is comparable to the $L_{p}$-norm of $f$ :

$$
\|S f\|_{p} \simeq\|f\|_{p}, \quad f \in L_{p}
$$

with constants depending only on $p$ and $k$. Moreover, for $p=1$, it is shown in [9] that

$$
\|S f\|_{1} \simeq \sup _{\varepsilon \in\{-1,1\}^{\mathbb{Z}}}\left\|\sum_{n} \varepsilon_{n} \Delta_{n} f\right\|_{1}, \quad S f \in L_{1},
$$

with constants depending only on $k$ and where the proof of the $\lesssim$-part only uses Khintchine's inequality whereas the proof of the $\gtrsim$-part uses fine properties of the functions $\Delta_{n} f$.

\section{$2.5 L_{p}\left(\ell_{q}\right)$-spaces}

For $1 \leq p, q \leq \infty$, we denote by $L_{p}\left(\ell_{q}\right)$ the space of sequences of measurable functions $\left(f_{n}\right)$ on $[0,1]$ so that the norm

$$
\left\|\left(f_{n}\right)\right\|_{L_{p}\left(\ell_{q}\right)}=\left(\int_{0}^{1}\left(\sum_{n}\left|f_{n}(t)\right|^{q}\right)^{p / q} \mathrm{~d} t\right)^{1 / p}
$$

is finite (with the obvious modifications if $p=\infty$ or $q=\infty$ ). For $1 \leq p, q<\infty$, the dual space (see [2]) of $L_{p}\left(\ell_{q}\right)$ is $L_{p^{\prime}}\left(\ell_{q^{\prime}}\right)$ with $p^{\prime}=p /(p-1), q^{\prime}=q /(q-1)$ and the duality pairing

$$
\left\langle\left(f_{n}\right),\left(g_{n}\right)\right\rangle=\int_{0}^{1} \sum_{n} f_{n}(t) g_{n}(t) \mathrm{d} t .
$$

Hölder's inequality takes the form $\left|\left\langle\left(f_{n}\right),\left(g_{n}\right)\right\rangle\right| \leq\left\|\left(f_{n}\right)\right\|_{L_{p}\left(\ell_{q}\right)}\left\|\left(g_{n}\right)\right\|_{L_{p^{\prime}}\left(\ell_{q^{\prime}}\right)}$.

\section{Main results}

In this section, we prove our main results. Section 3.1 defines and gives properties of suitable positive operators that dominate our (non-positive) operators $P_{n}=P_{n}^{(k)}$ pointwise. In Sect. 3.2, we use those operators to give a spline version of Stein's inequality (1.2). A useful property of conditional expectations is the tower property $\mathbb{E}_{\mathscr{G}} \mathbb{E}_{\mathscr{F}} f=\mathbb{E}_{\mathscr{G}} f$ for $\mathscr{G} \subset \mathscr{F}$. In this form, it extends to the operators $\left(P_{n}\right)$, but not to the operators $T$ from Sect. 3.1. In Sect. 3.3 we prove a version of the tower property for those operators. Section 3.4 is devoted to establishing a duality estimate using a spline square function, which is the crucial ingredient in the proofs of the spline versions of both Lépingle's inequality (1.1) and $H_{1}$-BMO duality in Sect. 4. 


\subsection{The positive operators $T$}

As above, let $\mathscr{F}$ be an interval $\sigma$-algebra on $[0,1],\left(N_{i}\right)$ the B-spline basis of $\mathscr{S}_{k}(\mathscr{F})$, $E_{i}$ the support of $N_{i}$ and $E_{i j}$ the smallest interval containing both $E_{i}$ and $E_{j}$. Moreover, let $q$ be a positive number smaller than 1 . Then, we define the linear operator $T=$ $T_{\mathscr{F}, q, k}$ by

$$
T f(x):=\sum_{i, j} \frac{q^{|i-j|}}{\left|E_{i j}\right|}\left\langle f, \mathbb{1}_{E_{i}}\right\rangle \mathbb{1}_{E_{j}}(x)=\int_{0}^{1} K(x, t) f(t) \mathrm{d} t,
$$

where the kernel $K=K_{T}$ is given by

$$
K(x, t)=\sum_{i, j} \frac{q^{|i-j|}}{\left|E_{i j}\right|} \mathbb{1}_{E_{i}}(t) \cdot \mathbb{1}_{E_{j}}(x) .
$$

We observe that the operator $T$ is selfadjoint (w.r.t the standard inner product on $\left.L_{2}\right)$ and

$$
k \leq K_{x}:=\int_{0}^{1} K(x, t) \mathrm{d} t \leq \frac{2(k+1)}{1-q}, \quad x \in[0,1],
$$

which, in particular, implies the boundedness of the operator $T$ on $L_{1}$ and $L_{\infty}$ :

$$
\|T f\|_{1} \leq \frac{2(k+1)}{1-q}\|f\|_{1}, \quad\|T f\|_{\infty} \leq \frac{2(k+1)}{1-q}\|f\|_{\infty} .
$$

Another very important property of $T$ is that it is a positive operator, i.e. it maps nonnegative functions to non-negative functions and that $T$ satisfies Jensen's inequality in the form

$$
\varphi(T f(x)) \leq K_{x}^{-1} T\left(\varphi\left(K_{x} \cdot f\right)\right)(x), \quad f \in L_{1}, x \in[0,1],
$$

for convex functions $\varphi$. This is seen by applying the classical Jensen inequality to the probability measure $K(t, x) \mathrm{d} t / K_{x}$.

Let $\mathscr{M} f$ denote the Hardy-Littlewood maximal function of $f \in L_{1}$, i.e.

$$
\mathscr{M} f(x)=\sup _{I \ni x} \frac{1}{|I|} \int_{I}|f(y)| \mathrm{d} y
$$

where the supremum is taken over all subintervals of $[0,1]$ that contain the point $x$. This operator is of weak type $(1,1)$, i.e.

$$
|\{\mathscr{M} f>\lambda\}| \leq C \lambda^{-1}\|f\|_{1}, \quad f \in L_{1}, \lambda>0
$$


for some constant $C$. Since trivially we have the estimate $\|\mathscr{M} f\|_{\infty} \leq\|f\|_{\infty}$, by Marcinkiewicz interpolation, for any $p>1$, there exists a constant $C_{p}$ depending only on $p$ so that

$$
\|\mathscr{M} f\|_{p} \leq C_{p}\|f\|_{p}
$$

For those assertions about $\mathscr{M}$, we refer to (for instance) [23].

The significance of $T$ and $\mathscr{M}$ at this point is that we can use formula (2.4) and estimate (2.5) to obtain the pointwise bound

$$
|P f(x)| \leq C_{1}(T|f|)(x) \leq C_{2} \mathscr{M}(x), \quad f \in L_{1}, x \in[0,1]
$$

where $T=T_{\mathscr{F}, q, k}$ with $q$ given by (2.5), $C_{1}$ is a constant that depends only on $k$ and $C_{2}$ is a constant that depends only on $k$ and the geometric progression $q$. But as the parameter $q<1$ in (2.5) depends only on $k$, the constant $C_{2}$ will also only depend on $k$.

In other words, (3.3) tells us that the positive operator $T$ dominates the non-positive operator $P$ pointwise, but at the same time, $T$ is dominated by the Hardy-Littlewood maximal function $\mathscr{M}$ pointwise and independently of $\mathscr{F}$.

\subsection{Stein's inequality for splines}

We now use this pointwise dominating, positive operator $T$ to prove Stein's inequality for spline projections. For this, let $\left(\mathscr{F}_{n}\right)$ be an interval filtration on $[0,1]$ and $P_{n}$ be the orthogonal projection operator onto the space $\mathscr{S}_{k}\left(\mathscr{F}_{n}\right)$ of splines of order $k$ corresponding to $\mathscr{F}_{n}$. Working with the positive operators $T_{\mathscr{F}_{n}, q, k}$ instead of the nonpositive operators $P_{n}$, the proof of Stein's inequality (1.2) for spline projections can be carried over from the martingale case (cf. [1,24]). For completeness, we include it here.

Theorem 3.1 Suppose that $\left(f_{n}\right)$ is a sequence of arbitrary integrable functions on $[0,1]$. Then, for $1 \leq r \leq p<\infty$ or $1<p \leq r \leq \infty$,

$$
\left\|\left(P_{n} f_{n}\right)\right\|_{L_{p}\left(\ell_{r}\right)} \lesssim\left\|\left(f_{n}\right)\right\|_{L_{p}\left(\ell_{r}\right)}
$$

where the implied constant depends only on $p, r$ and $k$.

Proof By (3.3), it suffices to prove this inequality for the operators $T_{n}=T_{\mathscr{F}_{n}, q, k}$ with $q$ given by (2.5) instead of the operators $P_{n}$. First observe that for $r=p=1$, the assertion follows from Shadrin's theorem ((i) on page 1). Inequality (3.3) and the $L_{p^{\prime}}$-boundedness of $\mathscr{M}$ for $1<p^{\prime} \leq \infty$ imply that

$$
\left\|\sup _{1 \leq n \leq N}\left|T_{n} f\right|\right\|_{p^{\prime}} \leq C_{p^{\prime}, k}\|f\|_{p^{\prime}}, \quad f \in L_{p^{\prime}}
$$

with a constant $C_{p^{\prime}, k}$ depending on $p^{\prime}$ and $k$. Let $1 \leq p<\infty$ and $U_{N}: L_{p}\left(\ell_{1}^{N}\right) \rightarrow L_{p}$ be given by $\left(g_{1}, \ldots, g_{N}\right) \mapsto \sum_{j=1}^{N} T_{j} g_{j}$. Inequality (3.5) implies the boundedness of 
the adjoint $U_{N}^{*}: L_{p^{\prime}} \rightarrow L_{p^{\prime}}\left(\ell_{\infty}^{N}\right), f \mapsto\left(T_{j} f\right)_{j=1}^{N}$ for $p^{\prime}=p /(p-1)$ by a constant independent of $N$ and therefore also the boundedness of $U_{N}$. Since $\left|T_{j} f\right| \leq T_{j}|f|$ by the positivity of $T_{j}$, letting $N \rightarrow \infty$ implies (3.4) for $T_{n}$ instead of $P_{n}$ in the case $r=1$ and outer parameter $1 \leq p<\infty$.

If $1<r \leq p$, we use Jensen's inequality (3.2) and estimate (3.1) to obtain

$$
\sum_{j=1}^{N}\left|T_{j} g_{j}\right|^{r} \lesssim \sum_{j=1}^{N} T_{j}\left(\left|g_{j}\right|^{r}\right)
$$

and apply the result for $r=1$ and the outer parameter $p / r$ to get the result for $1 \leq r \leq p<\infty$. The cases $1<p \leq r \leq \infty$ now just follow from this result using duality and the self-adjointness of $T_{j}$.

\subsection{Tower property of $T$}

Next, we will prove a substitute of the tower property $\mathbb{E}_{\mathscr{G}} \mathbb{E}_{\mathscr{F}} f=\mathbb{E}_{\mathscr{G}} f(\mathscr{G} \subset \mathscr{F})$ for conditional expectations that applies to the operators $T$.

To formulate this result, we need a suitable notion of regularity for $\sigma$-algebras which we now describe. Let $\mathscr{F}$ be an interval $\sigma$-algebra, let $\left(N_{j}\right)$ be the B-spline basis of $\mathscr{S}_{k}(\mathscr{F})$ and denote by $E_{j}$ the support of the function $N_{j}$. The $k$-regularity parameter $\gamma_{k}(\mathscr{F})$ is defined as

$$
\gamma_{k}(\mathscr{F}):=\max _{i} \max \left(\left|E_{i}\right| /\left|E_{i+1}\right|,\left|E_{i+1}\right| /\left|E_{i}\right|\right)
$$

where the first maximum is taken over all $i$ so that $E_{i}$ and $E_{i+1}$ are defined. The name $k$-regularity is motivated by the fact that each B-spline support $E_{i}$ of order $k$ consists of at most $k$ (neighboring) atoms of the $\sigma$-algebra $\mathscr{F}$.

Proposition 3.2 (Tower property of $T$ ) Let $\mathscr{G} \subset \mathscr{F}$ be two interval $\sigma$-algebras on $[0,1]$. Let $S=T_{\mathscr{G}, \sigma, k}$ and $T=T_{\mathscr{F}, \tau, k^{\prime}}$ for some $\sigma, \tau \in(0,1)$ and some positive integers $k, k^{\prime}$. Then, for all $q>\max (\tau, \sigma)$, there exists a constant $C$ depending on $q, k, k^{\prime}$ so that

$$
|S T f(x)| \leq C \cdot \gamma^{k} \cdot\left(T_{\mathscr{G}, q, k}|f|\right)(x), \quad f \in L_{1}, x \in[0,1],
$$

where $\gamma=\gamma_{k}(\mathscr{G})$ denotes the $k$-regularity parameter of $\mathscr{G}$.

Proof Let $\left(F_{i}\right)$ be the collection of B-spline supports in $\mathscr{S}_{k^{\prime}}(\mathscr{F})$ and $\left(G_{i}\right)$ the collection of B-spline supports in $\mathscr{S}_{k}(\mathscr{G})$. Moreover, we denote by $F_{i j}$ the smallest interval containing $F_{i}$ and $F_{j}$ and by $G_{i j}$ the smallest interval containing $G_{i}$ and $G_{j}$.

We show (3.6) by showing the following inequality for the kernels $K_{S}$ of $S$ and $K_{T}$ of $T$ (cf. 3.1)

$$
\int_{0}^{1} K_{S}(x, t) K_{T}(t, s) \mathrm{d} t \leq C \gamma^{k} \sum_{i, j} \frac{q^{|i-j|}}{\left|G_{i j}\right|} \mathbb{1}_{G_{i}}(x) \mathbb{1}_{G_{j}}(s), \quad x, s \in[0,1]
$$


for all $q>\max (\tau, \sigma)$ and some constant $C$ depending on $q, k, k^{\prime}$. In order to prove this inequality, we first fix $x, s \in[0,1]$ and choose $i$ such that $x \in G_{i}$ and $\ell$ such that $s \in F_{\ell}$. Moreover, based on $\ell$, we choose $j$ so that $s \in G_{j}$ and $G_{j} \supset F_{\ell}$. There are at most $\max \left(k, k^{\prime}\right)$ choices for each of the indices $i, \ell, j$ and without restriction, we treat those choices separately, i.e. we only have to estimate the expression

$$
\sum_{m, r} \frac{\sigma^{|m-i|} \tau^{|r-\ell|}\left|G_{m} \cap F_{r}\right|}{\left|G_{i m}\right|\left|F_{\ell r}\right|} .
$$

Since, for each $r$, there are also at most $k+k^{\prime}-1$ indices $m$ so that $\left|G_{m} \cap F_{r}\right|>0$ (recall that $\mathscr{G} \subset \mathscr{F}$ ), we choose one such index $m=m(r)$ and estimate

$$
\Sigma=\sum_{r} \frac{\sigma^{|m(r)-i|} \tau^{|r-\ell|}\left|G_{m(r)} \cap F_{r}\right|}{\left|G_{i, m(r)}\right|\left|F_{\ell r}\right|} .
$$

Now, observe that for any parameter choice of $r$ in the above sum,

$$
G_{i, m(r)} \cup F_{\ell r} \supseteq\left(G_{i j} \backslash G_{j}\right) \cup G_{i}
$$

and therefore, since also $G_{m(r)} \cap F_{r} \subset G_{i, m(r)} \cap F_{\ell r}$,

$$
\Sigma \leq \frac{2}{\left|\left(G_{i j} \backslash G_{j}\right) \cup G_{i}\right|} \sum_{r} \sigma^{|m(r)-i|} \tau^{|r-\ell|},
$$

which, using the $k$-regularity parameter $\gamma=\gamma_{k}(\mathscr{G})$ of the $\sigma$-algebra $\mathscr{G}$ and denoting $\lambda=\max (\tau, \sigma)$, we estimate by

$$
\begin{aligned}
\Sigma & \leq \frac{2 \gamma^{k}}{\left|G_{i j}\right|} \sum_{m} \lambda^{|m-i|} \sum_{r: m(r)=m} \lambda^{|r-\ell|} \lesssim \frac{\gamma^{k}}{\left|G_{i j}\right|} \sum_{m} \lambda^{|i-m|+|m-j|} \\
& \lesssim \frac{\gamma^{k}}{\left|G_{i j}\right|}(|i-j|+1) \lambda^{|i-j|},
\end{aligned}
$$

where the implied constants depend on $\lambda, k, k^{\prime}$ and the estimate $\sum_{r: m(r)=m} \lambda^{|r-\ell|} \lesssim$ $\lambda^{|m-j|}$ used the fact that, essentially, there are more atoms of $\mathscr{F}$ between $F_{r}$ and $F_{\ell}$ (for $r$ as in the sum) than atoms of $\mathscr{G}$ between $G_{m}$ and $G_{j}$. Finally, we see that for any $q>\lambda$,

$$
\Sigma \lesssim C \gamma^{k} \frac{q^{|i-j|}}{\left|G_{i j}\right|}
$$

for some constant $C$ depending on $q, k, k^{\prime}$, and, as $x \in G_{i}$ and $s \in G_{j}$, this shows inequality (3.7).

As a corollary of Proposition 3.2, we have 
Corollary 3.3 Let $\left(f_{n}\right)$ be functions in $L_{1}$. We denote by $P_{n}$ the orthogonal projection onto $\mathscr{S}_{k}\left(\mathscr{F}_{n}\right)$ and by $P_{n}^{\prime}$ the orthogonal projection onto $\mathscr{S}_{k^{\prime}}\left(\mathscr{F}_{n}\right)$ for some positive integers $k, k^{\prime}$. Moreover, let $T_{n}$ be the operator $T_{\mathscr{F}_{n}, q, k}$ from (3.3) dominating $P_{n}$ pointwise.

Then, for any integer $n$ and for any $1 \leq p \leq \infty$,

$$
\left\|\sum_{\ell \geq n} P_{n}\left(\left(P_{\ell-1}^{\prime} f_{\ell}\right)^{2}\right)\right\|_{p} \lesssim\left\|\sum_{\ell \geq n} T_{n}\left(\left(P_{\ell-1}^{\prime} f_{\ell}\right)^{2}\right)\right\|_{p} \lesssim \gamma_{k}\left(\mathscr{F}_{n}\right)^{k} \cdot\left\|\sum_{\ell \geq n} f_{\ell}^{2}\right\|_{p}
$$

where the implied constants only depend on $k$ and $k^{\prime}$.

We remark that by Jensen's inequality and the tower property, this is trivial for conditional expectations $\mathbb{E}\left(\cdot \mid \mathscr{F}_{n}\right)$ instead of the operators $P_{n}, T_{n}, P_{\ell-1}^{\prime}$ even with an absolute constant on the right hand side.

Proof We denote by $T_{n}$ the operator $T_{\mathscr{F}_{n}, q, k}$ and by $T_{n}^{\prime}$ the operator $T_{\mathscr{F}_{n}, q^{\prime}, k^{\prime}}$, where the parameters $q, q^{\prime}<1$ are given by inequality (3.3) depending on $k$ and $k^{\prime}$ respectively. Setting $U_{n}:=T_{\mathscr{F}_{n}, \max \left(q, q^{\prime}\right)^{1 / 2}, k}$, we perform the following chain of inequalities, where we use the positivity of $T_{n}$ and (3.3), Jensen's inequality for $T_{\ell-1}^{\prime}$, the tower property for $T_{n} T_{\ell-1}^{\prime}$ and the $L_{p}$-boundedness of $U_{n}$, respectively:

$$
\begin{aligned}
\left\|\sum_{\ell \geq n} T_{n}\left(\left(P_{\ell-1}^{\prime} f_{\ell}\right)^{2}\right)\right\|_{p} & \lesssim\left\|\sum_{\ell \geq n} T_{n}\left(\left(T_{\ell-1}^{\prime}\left|f_{\ell}\right|\right)^{2}\right)\right\|_{p} \\
& \lesssim\left\|\sum_{\ell \geq n} T_{n}\left(T_{\ell-1}^{\prime} f_{\ell}^{2}\right)\right\|_{p} \\
& \leq\left\|T_{n}\left(T_{n-1}^{\prime} f_{n}^{2}\right)\right\|_{p}+\left\|\sum_{\ell>n} T_{n}\left(T_{\ell-1}^{\prime} f_{\ell}^{2}\right)\right\|_{p} \\
& \lesssim\left\|f_{n}^{2}\right\|_{p}+\gamma_{k}\left(\mathscr{F}_{n}\right)^{k} \cdot\left\|\sum_{\ell>n} U_{n}\left(f_{\ell}^{2}\right)\right\|_{p} \\
& \lesssim \gamma_{k}\left(\mathscr{F}_{n}\right)^{k} \cdot\left\|\sum_{\ell \geq n} f_{\ell}^{2}\right\|_{p}
\end{aligned}
$$

where the implied constants only depend on $k$ and $k^{\prime}$.

\subsection{A duality estimate using a spline square function}

In order to give the desired duality estimate contained in Theorem 3.6, we need the following construction of a function $g_{n} \in \mathscr{S}_{k}\left(\mathscr{F}_{n}\right)$ based on a spline square function.

Proposition 3.4 Let $\left(f_{n}\right)$ be a sequence of functions with $f_{n} \in \mathscr{S}_{k}\left(\mathscr{F}_{n}\right)$ for all $n$ and set

$$
X_{n}:=\sum_{\ell \leq n} f_{\ell}^{2}
$$


Then, there exists a sequence of non-negative functions $g_{n} \in \mathscr{S}_{k}\left(\mathscr{F}_{n}\right)$ so that for each $n$,

(1) $g_{n} \leq g_{n+1}$,

(2) $X_{n}^{1 / 2} \leq g_{n}$

(3) $\mathbb{E} g_{n} \lesssim \mathbb{E} X_{n}^{1 / 2}$, where the implied constant depends on $k$ and on $\sup _{m \leq n} \gamma_{k}\left(\mathscr{F}_{m}\right)$.

For the proof of this result, we need the following simple lemma.

Lemma 3.5 Let $c_{1}$ be a positive constant and let $\left(A_{j}\right)_{j=1}^{N}$ be a sequence of atoms in $\mathscr{F}_{n}$. Moreover, let $\ell:\{1, \ldots, N\} \rightarrow\{1, \ldots, n\}$ and, for each $j \in\{1, \ldots, N\}$, let $B_{j}$ be a subset of an atom $D_{j}$ of $\mathscr{F}_{\ell(j)}$ with

$$
\left|B_{j}\right| \geq c_{1} \sum_{\substack{i: \ell(i) \geq \ell(j), D_{i} \subseteq D_{j}}}\left|A_{i}\right| .
$$

Then, there exists a map $\varphi$ on $\{1, \ldots, N\}$ so that

(1) $|\varphi(j)|=c_{1}\left|A_{j}\right|$ for all $j$,

(2) $\varphi(j) \subseteq B_{j}$ for all $j$,

(3) $\varphi(i) \cap \varphi(j)=\emptyset$ for all $i \neq j$.

Proof Without restriction, we assume that the sequence $\left(A_{j}\right)$ is enumerated such that $\ell(j+1) \leq \ell(j)$ for all $1 \leq j \leq N-1$. We first choose $\varphi(1)$ as an arbitrary (measurable) subset of $B_{1}$ with measure $c_{1}\left|A_{1}\right|$, which is possible by assumption (3.8). Next, we assume that for $1 \leq j \leq j_{0}$, we have constructed $\varphi(j)$ with the properties

(1) $|\varphi(j)|=c_{1}\left|A_{j}\right|$,

(2) $\varphi(j) \subseteq B_{j}$,

(3) $\varphi(j) \cap \cup_{i<j} \varphi(i)=\emptyset$.

Based on that, we now construct $\varphi\left(j_{0}+1\right)$. Define the index sets $\Gamma=\{i: \ell(i) \geq$ $\left.\ell\left(j_{0}+1\right), D_{i} \subseteq D_{j_{0}+1}\right\}$ and $\Lambda=\left\{i: i \leq j_{0}+1, D_{i} \subseteq D_{j_{0}+1}\right\}$. Since we assumed that $\ell$ is decreasing, we have $\Lambda \subseteq \Gamma$ and by the nestedness of the $\sigma$-algebras $\mathscr{F}_{n}$, we have for $i \leq j_{0}+1$ that either $D_{i} \subset D_{j_{0}+1}$ or $\left|D_{i} \cap D_{j_{0}+1}\right|=0$. This implies

$$
\begin{aligned}
\left|B_{j_{0}+1} \backslash \bigcup_{i \leq j_{0}} \varphi(i)\right| & =\left|B_{j_{0}+1}\right|-\left|B_{j_{0}+1} \cap \bigcup_{i \leq j_{0}} \varphi(i)\right| \\
& \geq c_{1} \sum_{i \in \Gamma}\left|A_{i}\right|-\left|D_{j_{0}+1} \cap \bigcup_{i \leq j_{0}} \varphi(i)\right| \\
& \geq c_{1} \sum_{i \in \Lambda}\left|A_{i}\right|-\left|\bigcup_{i \in \Lambda \backslash\left\{j_{0}+1\right\}} \varphi(i)\right| \\
& \geq c_{1} \sum_{i \in \Lambda}\left|A_{i}\right|-\sum_{i \in \Lambda \backslash\left\{j_{0}+1\right\}} c_{1}\left|A_{i}\right|=c_{1}\left|A_{j_{0}+1}\right| .
\end{aligned}
$$

Therefore, we can choose $\varphi\left(j_{0}+1\right) \subseteq B_{j_{0}+1}$ that is disjoint to $\varphi(i)$ for any $i \leq j_{0}$ and $\left|\varphi\left(j_{0}+1\right)\right|=c_{1}\left|A_{j_{0}+1}\right|$ which completes the proof. 
Proof of Proposition 3.4 Fix $n$ and let $\left(N_{n, j}\right)$ be the B-spline basis of $\mathscr{S}_{k}\left(\mathscr{F}_{n}\right)$. Moreover, for any $j$, set $E_{n, j}=\operatorname{supp} N_{n, j}$ and $a_{n, j}:=\max _{\ell \leq n} \max _{r: E_{\ell, r} \supset E_{n, j}}\left\|X_{\ell}\right\|_{L_{\infty}\left(E_{\ell, r}\right)}^{1 / 2}$ and we define $\ell(j) \leq n$ and $r(j)$ so that $E_{\ell(j), r(j)} \supseteq E_{n, j}$ and $a_{n, j}=$ $\left\|X_{\ell(j)}\right\|_{L_{\infty}\left(E_{\ell(j), r(j)}\right)}^{1 / 2}$. Set

$$
g_{n}:=\sum_{j} a_{n, j} N_{n, j} \in \mathscr{S}_{k}\left(\mathscr{F}_{n}\right)
$$

and it will be proved subsequently that this $g_{n}$ has the desired properties.

PROPERTY (1): In order to show $g_{n} \leq g_{n+1}$, we use Theorem 2.4 to write

$$
g_{n}=\sum_{j} a_{n, j} N_{n, j}=\sum_{j} \beta_{n, j} N_{n+1, j}
$$

where $\beta_{n, j}$ is a convex combination of those $a_{n, r}$ with $E_{n+1, j} \subseteq E_{n, r}$, and thus

$$
g_{n} \leq \sum_{j}\left(\max _{r: E_{n+1, j} \subseteq E_{n, r}} a_{n, r}\right) N_{n+1, j}
$$

By the very definition of $a_{n+1, j}$, we have

$$
\max _{r: E_{n+1, j} \subseteq E_{n, r}} a_{n, r} \leq a_{n+1, j}
$$

and therefore, $g_{n} \leq g_{n+1}$ pointwise, since the B-splines $\left(N_{n+1, j}\right)_{j}$ are nonnegative functions.

PROPERTY (2): Now we show that $X_{n}^{1 / 2} \leq g_{n}$. Indeed, for any $x \in[0,1]$,

$$
g_{n}(x)=\sum_{j} a_{n, j} N_{n, j}(x) \geq \min _{j: E_{n, j} \ni x} a_{n, j} \geq \min _{j: E_{n, j} \ni x}\left\|X_{n}\right\|_{L_{\infty}\left(E_{n, j}\right)}^{1 / 2} \geq X_{n}(x)^{1 / 2}
$$

since the collection of B-splines $\left(N_{n, j}\right)_{j}$ forms a partition of unity.

PROPERTY (3): Finally, we show $\mathbb{E} g_{n} \lesssim \mathbb{E} X_{n}^{1 / 2}$, where the implied constant depends only on $k$ and on $\sup _{m \leq n} \gamma_{k}\left(\mathscr{F}_{m}\right)$. By B-spline stability (Theorem 2.3), we estimate the integral of $g_{n}$ by

$$
\mathbb{E} g_{n} \lesssim \sum_{j}\left|E_{n, j}\right| \cdot\left\|X_{\ell(j)}\right\|_{L_{\infty}\left(E_{\ell(j), r(j)}\right)}^{1 / 2}
$$

where the implied constant only depends on $k$. In order to continue the estimate, we next show the inequality

$$
\left\|X_{\ell}\right\|_{L_{\infty}\left(E_{\ell, r}\right)} \lesssim \max _{s:\left|E_{\ell, r} \cap E_{\ell, s}\right|>0}\left\|X_{\ell}\right\|_{L_{\infty}\left(J_{\ell, s}\right)}
$$


where by $J_{\ell, s}$ we denote an atom of $\mathscr{F}_{\ell}$ with $J_{\ell, s} \subset E_{\ell, s}$ of maximal length and the implied constant depends only on $k$. Indeed, we use Theorem 2.3 in the form of (2.3) to get $\left(f_{m} \in \mathscr{S}_{k}\left(\mathscr{F}_{\ell}\right)\right.$ for $\left.m \leq \ell\right)$

$$
\begin{aligned}
\left\|X_{\ell}\right\|_{L_{\infty}\left(E_{\ell, r}\right)} & \leq \sum_{m \leq \ell}\left\|f_{m}\right\|_{L_{\infty}\left(E_{\ell, r}\right)}^{2} \\
& \lesssim \sum_{m \leq \ell s:\left|E_{\ell, s} \cap E_{\ell, r}\right|>0} \sum_{m}\left\|f_{m}\right\|_{L_{\infty}\left(J_{\ell, s}\right)}^{2}=\sum_{s:\left|E_{\ell, s} \cap E_{\ell, r}\right|>0} \sum_{m \leq \ell}\left\|f_{m}\right\|_{L_{\infty}\left(J_{\ell, s}\right)}^{2} .
\end{aligned}
$$

Now observe that for atoms $I$ of $\mathscr{F}_{\ell}$, due to the equivalence of $p$-norms of polynomials (cf. Corollary 2.2),

$$
\sum_{m \leq \ell}\left\|f_{m}\right\|_{L_{\infty}(I)}^{2} \lesssim \sum_{m \leq \ell} \frac{1}{|I|} \int_{I} f_{m}^{2}=\frac{1}{|I|} \int_{I} X_{\ell} \leq\left\|X_{\ell}\right\|_{L_{\infty}(I)},
$$

which means that, inserting this in estimate (3.11),

$$
\left\|X_{\ell}\right\|_{L_{\infty}\left(E_{\ell, r}\right)} \lesssim \sum_{s:\left|E_{\ell, s} \cap E_{\ell, r}\right|>0}\left\|X_{\ell}\right\|_{L_{\infty}\left(J_{\ell, s}\right)}
$$

and, since there are at most $k$ indices $s$ so that $\left|E_{\ell, s} \cap E_{\ell, r}\right|>0$, we have established (3.10).

We define $K_{\ell, r}$ to be an interval $J_{\ell, s}$ with $\left|E_{\ell, r} \cap E_{\ell, s}\right|>0$ so that

$$
\max _{s:\left|E_{\ell, r} \cap E_{\ell, s}\right|>0}\left\|X_{\ell}\right\|_{L_{\infty}\left(J_{\ell, s}\right)}=\left\|X_{\ell}\right\|_{L_{\infty}\left(K_{\ell, r}\right)} .
$$

This means, after combining (3.9) with (3.10), we have

$$
\mathbb{E} g_{n} \lesssim \sum_{j}\left|J_{n, j}\right| \cdot\left\|X_{\ell(j)}\right\|_{L_{\infty}\left(K_{\ell(j), r(j)}\right)}^{1 / 2}
$$

We now apply Lemma 3.5 with the function $\ell$ and the choices

$$
\begin{aligned}
& A_{j}=J_{n, j}, \quad D_{j}=K_{\ell(j), r(j)}, \\
& B_{j}=\left\{t \in D_{j}: X_{\ell(j)}(t) \geq 8^{-2(k-1)}\left\|X_{\ell(j)}\right\|_{L_{\infty}\left(D_{j}\right)}\right\} .
\end{aligned}
$$

In order to see Assumption (3.8) of Lemma 3.5, fix the index $j$ and let $i$ be such that $\ell(i) \geq \ell(j)$. By definition of $D_{i}=K_{\ell(i), r(i)}$, the smallest interval containing $J_{n, i}$ and $D_{i}$ contains at most $2 k-1$ atoms of $\mathscr{F}_{\ell(i)}$ and, if $D_{i} \subset D_{j}$, the smallest interval containing $J_{n, i}$ and $D_{j}$ contains at most $2 k-1$ atoms of $\mathscr{F}_{\ell(j)}$. This means that, in particular, $J_{n, i}$ is a subset of the union $V$ of $4 k$ atoms of $\mathscr{F}_{\ell(j)}$ with $D_{j} \subset V$. Since 
each atom of $\mathscr{F}_{n}$ occurs at most $k$ times in the sequence $\left(A_{j}\right)$, there exists a constant $c_{1}$ depending on $k$ and $\sup _{u \leq \ell(j)} \gamma_{k}\left(\mathscr{F}_{u}\right) \leq \sup _{u \leq n} \gamma_{k}\left(\mathscr{F}_{u}\right)$ so that

$$
\left|D_{j}\right| \geq c_{1} \sum_{\substack{i: \ell(i) \geq \ell(j) \\ D_{i} \subset D_{j}}}\left|A_{i}\right|
$$

which, since $\left|B_{j}\right| \geq\left|D_{j}\right| / 2$ by Corollary 2.2, shows that the assumption of Lemma 3.5 holds true and we get a function $\varphi$ so that $|\varphi(j)|=c_{1}\left|J_{n, j}\right| / 2, \varphi(j) \subset B_{j}$, $\varphi(i) \cap \varphi(j)=\emptyset$ for all $i, j$. Using these properties of $\varphi$, we continue the estimate in (3.12) and write

$$
\begin{aligned}
\mathbb{E} g_{n} & \lesssim \sum_{j}\left|J_{n, j}\right| \cdot\left\|X_{\ell(j)}\right\|_{L_{\infty}\left(D_{j}\right)}^{1 / 2} \leq 8^{k-1} \cdot \sum_{j} \frac{\left|J_{n, j}\right|}{|\varphi(j)|} \int_{\varphi(j)} X_{\ell(j)}^{1 / 2} \\
& =\frac{2}{c_{1}} \cdot 8^{k-1} \cdot \sum_{j} \int_{\varphi(j)} X_{\ell(j)}^{1 / 2} \\
& \lesssim \sum_{j} \int_{\varphi(j)} X_{n}^{1 / 2} \leq \mathbb{E} X_{n}^{1 / 2}
\end{aligned}
$$

with constants depending only on $k$ and $\sup _{u \leq n} \gamma_{k}\left(\mathscr{F}_{u}\right)$.

Employing this construction of $g_{n}$, we now give the following duality estimate for spline projections (for the martingale case, see for instance [8]). The martingale version of this result is the essential estimate in the proof of both Lépingle's inequality (1.1) and the $H^{1}$-BMO duality.

Theorem 3.6 Let $\left(\mathscr{F}_{n}\right)$ be such that $\gamma:=\sup _{n} \gamma_{k}\left(\mathscr{F}_{n}\right)<\infty$ and $\left(f_{n}\right)_{n \geq 1}$ a sequence of functions with $f_{n} \in \mathscr{S}_{k}\left(\mathscr{F}_{n}\right)$ for each $n$. Additionally, let $h_{n} \in L_{1}$ be arbitrary. Then, for any $N$,

$$
\sum_{n \leq N} \mathbb{E}\left[\left|f_{n} \cdot h_{n}\right|\right] \lesssim \sqrt{2} \cdot \mathbb{E}\left[\left(\sum_{\ell \leq N} f_{\ell}^{2}\right)^{1 / 2}\right] \cdot \sup _{n \leq N}\left\|P_{n}\left(\sum_{\ell=n}^{N} h_{\ell}^{2}\right)\right\|_{\infty}^{1 / 2},
$$

where the implied constant is the same constant that appears in (3) of Proposition 3.4 and therefore only depends on $k$ and $\gamma$.

Proof Let $X_{n}:=\sum_{\ell \leq n} f_{\ell}^{2}$ and $f_{\ell} \equiv 0$ for $\ell>N$ and $\ell \leq 0$. By Proposition 3.4, we choose an increasing sequence $\left(g_{n}\right)$ of functions with $g_{0}=0, g_{n} \in \mathscr{S}_{k}\left(\mathscr{F}_{n}\right)$ and the properties $X_{n}^{1 / 2} \leq g_{n}$ and $\mathbb{E} g_{n} \lesssim \mathbb{E} X_{n}^{1 / 2}$ where the implied constant depends only on $k$ and $\gamma$. Then, apply Cauchy-Schwarz inequality by introducing the factor $g_{n}^{1 / 2}$ to get 


$$
\begin{aligned}
\sum_{n} \mathbb{E}\left[\left|f_{n} \cdot h_{n}\right|\right] & =\sum_{n} \mathbb{E}\left[\left|\frac{f_{n}}{g_{n}^{1 / 2}} \cdot g_{n}^{1 / 2} h_{n}\right|\right] \\
& \leq\left[\sum_{n} \mathbb{E}\left[f_{n}^{2} / g_{n}\right]\right]^{1 / 2} \cdot\left[\sum_{n} \mathbb{E}\left[g_{n} h_{n}^{2}\right]\right]^{1 / 2} .
\end{aligned}
$$

We estimate each of the factors on the right hand side separately and set

$$
\Sigma_{1}:=\sum_{n} \mathbb{E}\left[f_{n}^{2} / g_{n}\right], \quad \Sigma_{2}:=\sum_{n} \mathbb{E}\left[g_{n} h_{n}^{2}\right]
$$

The first factor is estimated by the pointwise inequality $X_{n}^{1 / 2} \leq g_{n}$ :

$$
\begin{aligned}
\Sigma_{1}=\mathbb{E}\left[\sum_{n} \frac{f_{n}^{2}}{g_{n}}\right] & \leq \mathbb{E}\left[\sum_{n} \frac{f_{n}^{2}}{X_{n}^{1 / 2}}\right] \\
& =\mathbb{E}\left[\sum_{n} \frac{X_{n}-X_{n-1}}{X_{n}^{1 / 2}}\right] \leq 2 \mathbb{E} \sum_{n}\left(X_{n}^{1 / 2}-X_{n-1}^{1 / 2}\right)=2 \mathbb{E} X_{N}^{1 / 2} .
\end{aligned}
$$

We continue with $\Sigma_{2}$ :

$$
\begin{aligned}
\Sigma_{2} & =\mathbb{E}\left[\sum_{\ell=1}^{N} g_{\ell} h_{\ell}^{2}\right]=\mathbb{E}\left[\sum_{\ell=1}^{N} \sum_{n=1}^{\ell}\left(g_{n}-g_{n-1}\right) h_{\ell}^{2}\right] \\
& =\mathbb{E}\left[\sum_{n=1}^{N}\left(g_{n}-g_{n-1}\right) \cdot \sum_{\ell=n}^{N} h_{\ell}^{2}\right] \\
& =\mathbb{E}\left[\sum_{n=1}^{N} P_{n}\left(g_{n}-g_{n-1}\right) \cdot \sum_{\ell=n}^{N} h_{\ell}^{2}\right] \\
& =\mathbb{E}\left[\sum_{n=1}^{N}\left(g_{n}-g_{n-1}\right) \cdot P_{n}\left(\sum_{\ell=n}^{N} h_{\ell}^{2}\right)\right] \\
& \leq \mathbb{E}\left[\sum_{n=1}^{N}\left(g_{n}-g_{n-1}\right)\right] \cdot \sup _{1 \leq n \leq N}\left\|P_{n}\left(\sum_{\ell=n}^{N} h_{\ell}^{2}\right)\right\|_{\infty},
\end{aligned}
$$

where the last inequality follows from $g_{n} \geq g_{n-1}$. Noting that by the properties of $g_{n}$, $\mathbb{E}\left[\sum_{n=1}^{N}\left(g_{n}-g_{n-1}\right)\right]=\mathbb{E} g_{N} \lesssim \mathbb{E} X_{N}^{1 / 2}$ and combining the two parts $\Sigma_{1}$ and $\Sigma_{2}$, we obtain the conclusion.

\section{Applications}

We give two applications of Theorem 3.6, (i) D. Lépingle's inequality and (ii) an analogue of C. Fefferman's $H_{1}$-BMO duality in the setting of splines. Once the results 
from Sect. 3 are known, the proofs of the subsequent results proceed similarly to their martingale counterparts in $[8,12]$ by using spline properties instead of martingale properties.

\subsection{Lépingle's inequality for splines}

Theorem 4.1 Let $k, k^{\prime}$ be positive integers. Let $\left(\mathscr{F}_{n}\right)$ be an interval filtration with $\sup _{n} \gamma_{k}\left(\mathscr{F}_{n}\right)<\infty$ and, for any $n, f_{n} \in \mathscr{S}_{k}\left(\mathscr{F}_{n}\right)$ and $P_{n}^{\prime}$ be the orthogonal projection operator on $\mathscr{S}_{k^{\prime}}\left(\mathscr{F}_{n}\right)$. Then,

$$
\left\|\left(P_{n-1}^{\prime} f_{n}\right)\right\|_{L_{1}\left(\ell_{2}\right)}=\left\|\left(\sum_{n}\left(P_{n-1}^{\prime} f_{n}\right)^{2}\right)^{1 / 2}\right\|_{1} \lesssim\left\|\left(\sum_{n} f_{n}^{2}\right)^{1 / 2}\right\|_{1}=\left\|\left(f_{n}\right)\right\|_{L_{1}\left(\ell_{2}\right)},
$$

where the implied constant depends only on $k, k^{\prime}$ and $\sup _{n} \gamma_{k}\left(\mathscr{F}_{n}\right)$.

We emphasize that the parameters $k$ and $k^{\prime}$ can be different here, $k$ being the spline order of the sequence $\left(f_{n}\right)$ and $k^{\prime}$ being the spline order of the projection operators $P_{n-1}^{\prime}$. In particular, the constant on the right hand side does not depend on the $k^{\prime}-$ regularity parameter $\sup _{n} \gamma_{k^{\prime}}\left(\mathcal{F}_{n}\right)$.

Proof We first assume that $f_{n}=0$ for $n>N$ and begin by using duality

$$
\mathbb{E}\left[\left(\sum_{n}\left(P_{n-1}^{\prime} f_{n}\right)^{2}\right)^{1 / 2}\right]=\sup _{\left(H_{n}\right)} \mathbb{E}\left[\sum_{n}\left(P_{n-1}^{\prime} f_{n}\right) \cdot H_{n}\right],
$$

where sup is taken over all $\left(H_{n}\right) \in L_{\infty}\left(\ell_{2}\right)$ with $\left\|\left(H_{n}\right)\right\|_{L_{\infty}\left(\ell_{2}\right)}=1$. By the selfadjointness of $P_{n-1}^{\prime}$,

$$
\mathbb{E}\left[\left(P_{n-1}^{\prime} f_{n}\right) \cdot H_{n}\right]=\mathbb{E}\left[f_{n} \cdot\left(P_{n-1}^{\prime} H_{n}\right)\right]
$$

Then we apply Theorem 3.6 for $f_{n}$ and $h_{n}=P_{n-1}^{\prime} H_{n}$ to obtain (denoting by $P_{n}$ the orthogonal projection operator onto $\left.\mathscr{S}_{k}\left(\mathscr{F}_{n}\right)\right)$

$$
\sum_{n \leq N}\left|\mathbb{E}\left[f_{n} \cdot h_{n}\right]\right| \lesssim \mathbb{E}\left[\left(\sum_{\ell \leq N} f_{\ell}^{2}\right)^{1 / 2}\right] \cdot \sup _{n \leq N}\left\|P_{n}\left(\sum_{\ell=n}^{N}\left(P_{\ell-1}^{\prime} H_{\ell}\right)^{2}\right)\right\|_{\infty}^{1 / 2}
$$

To estimate the right hand side, we note that for any $n$, by Corollary 3.3,

$$
\left\|P_{n}\left(\sum_{\ell=n}^{N}\left(P_{\ell-1}^{\prime} H_{\ell}\right)^{2}\right)\right\|_{\infty} \lesssim\left\|\sum_{\ell=n}^{N} H_{\ell}^{2}\right\|_{\infty}
$$


Therefore, (4.1) implies

$\mathbb{E}\left[\left(\sum_{n}\left(P_{n-1}^{\prime} f_{n}\right)^{2}\right)^{1 / 2}\right]=\sup _{\left(H_{n}\right)} \mathbb{E}\left[\sum_{n} f_{n} \cdot\left(P_{n-1}^{\prime} H_{n}\right)\right] \lesssim \mathbb{E}\left[\left(\sum_{\ell \leq N} f_{\ell}^{2}\right)^{1 / 2}\right]$

with a constant depending only on $k, k^{\prime}$ and $\sup _{n \leq N} \gamma_{k}\left(\mathscr{F}_{n}\right)$. Letting $N$ tend to infinity, we obtain the conclusion.

\section{2 $H_{1}-\mathrm{BMO}$ duality for splines}

We fix an interval filtration $\left(\mathscr{F}_{n}\right)_{n=1}^{\infty}$, a spline order $k$ and the orthogonal projection operators $P_{n}$ onto $\mathscr{S}_{k}\left(\mathscr{F}_{n}\right)$ and additionally, we set $P_{0}=0$. For $f \in L_{1}$, we introduce the notation

$$
\Delta_{n} f:=P_{n} f-P_{n-1} f, \quad S_{n}(f):=\left(\sum_{\ell=1}^{n}\left(\Delta_{\ell} f\right)^{2}\right)^{1 / 2}, \quad S(f)=\sup _{n} S_{n}(f) .
$$

Observe that for $\ell<n$ and $f, g \in L_{1}$,

$$
\mathbb{E}\left[\Delta_{\ell} f \cdot \Delta_{n} g\right]=\mathbb{E}\left[P_{\ell}\left(\Delta_{\ell} f\right) \cdot \Delta_{n} g\right]=\mathbb{E}\left[\Delta_{\ell} f \cdot P_{\ell}\left(\Delta_{n} g\right)\right]=0 .
$$

Let $V$ be the $L_{1}$-closure of $\cup_{n} \mathscr{S}_{k}\left(\mathscr{F}_{n}\right)$. Then, the uniform boundedness of $P_{n}$ on $L_{1}$ implies that $P_{n} f \rightarrow f$ in $L_{1}$ for $f \in V$. Next, set

$$
H_{1, k}=H_{1, k}\left(\left(\mathscr{F}_{n}\right)\right)=\{f \in V: \mathbb{E}(S(f))<\infty\}
$$

and equip $H_{1, k}$ with the norm $\|f\|_{H_{1, k}}=\mathbb{E} S(f)$. Define

$$
\mathrm{BMO}_{k}=\mathrm{BMO}_{k}\left(\left(\mathscr{F}_{n}\right)\right)=\left\{f \in V: \sup _{n}\left\|\sum_{\ell \geq n} T_{n}\left(\left(\Delta_{\ell} f\right)^{2}\right)\right\|_{\infty}<\infty\right\}
$$

and the corresponding quasinorm

$$
\|f\|_{\mathrm{BMO}_{k}}=\sup _{n}\left\|\sum_{\ell \geq n} T_{n}\left(\left(\Delta_{\ell} f\right)^{2}\right)\right\|_{\infty}^{1 / 2}
$$

where $T_{n}$ is the operator from (3.3) that dominates $P_{n}$ pointwise.

Let us now assume $\sup _{n} \gamma_{k}\left(\mathscr{F}_{n}\right)<\infty$. In this case we identify, similarly to $H_{1}$ BMO-duality (cf. [7,8,10]), $\mathrm{BMO}_{k}$ as the dual space of $H_{1, k}$. 
First, we use the duality estimate Theorem 3.6 and (4.2) to prove, for $f \in H_{1, k}$ and $h \in \mathrm{BMO}_{k}$,

$$
\left|\mathbb{E}\left[\left(P_{n} f\right) \cdot\left(P_{n} h\right)\right]\right| \leq \sum_{\ell \leq n} \mathbb{E}\left[\left|\Delta_{\ell} f\right| \cdot\left|\Delta_{\ell} h\right|\right] \lesssim\left\|S_{n}(f)\right\|_{1} \cdot\|h\|_{\mathrm{BMO}_{k}}
$$

This estimate also implies that the $\operatorname{limit}_{n} \lim _{n} \mathbb{E}\left[\left(P_{n} f\right) \cdot\left(P_{n} h\right)\right]$ exists and satisfies

$$
\left|\lim _{n} \mathbb{E}\left[\left(P_{n} f\right) \cdot\left(P_{n} h\right)\right]\right| \lesssim\|f\|_{H_{1, k}} \cdot\|h\|_{\mathrm{BMO}_{k}}
$$

On the other hand, similarly to the martingale case (see [8]), given a continuous linear functional $L$ on $H_{1, k}$, we extend $L$ norm-preservingly to a continuous linear functional $\Lambda$ on $L_{1}\left(\ell_{2}\right)$, which, by Sect. 2.5 , has the form

$$
\Lambda(\eta)=\mathbb{E}\left[\sum_{\ell} \sigma_{\ell} \eta_{\ell}\right], \quad \eta \in L_{1}\left(\ell_{2}\right)
$$

for some $\sigma \in L_{\infty}\left(\ell_{2}\right)$. The $k$-martingale spline sequence $h_{n}=\sum_{\ell<n} \Delta_{\ell} \sigma_{\ell}$ is bounded in $L_{2}$ and therefore, by the spline convergence theorem ((v) on page 2), has a limit $h \in L_{2}$ with $P_{n} h=h_{n}$ and which is also contained in $\mathrm{BMO}_{k}$. Indeed, by using Corollary 3.3, we obtain $\|h\|_{\mathrm{BMO}_{k}} \lesssim\|\sigma\|_{L_{\infty}\left(\ell_{2}\right)}=\|\Lambda\|=\|L\|$ with a constant depending only on $k$ and $\sup _{n} \gamma_{k}\left(\mathscr{F}_{n}\right)$. Moreover, for $f \in H_{1, k}$, since $L$ is continuous on $H_{1, k}$,

$$
\begin{aligned}
L(f)=\lim _{n} L\left(P_{n} f\right) & =\lim _{n} \Lambda\left(\left(\Delta_{1} f, \ldots, \Delta_{n} f, 0,0, \ldots\right)\right) \\
& =\lim _{n} \sum_{\ell=1}^{n} \mathbb{E}\left[\sigma_{\ell} \cdot \Delta_{\ell} f\right]=\lim _{n} \mathbb{E}\left[\left(P_{n} f\right) \cdot\left(P_{n} h\right)\right] .
\end{aligned}
$$

This yields the following

Theorem 4.2 If $\sup _{n} \gamma_{k}\left(\mathscr{F}_{n}\right)<\infty$, the linear mapping

$$
u: \mathrm{BMO}_{k} \rightarrow H_{1, k}^{*}, \quad h \mapsto\left(f \mapsto \lim _{n} \mathbb{E}\left[\left(P_{n} f\right) \cdot\left(P_{n} h\right)\right]\right)
$$

is bijective and satisfies

$$
\|u(h)\|_{H_{1, k}^{*}} \simeq\|h\|_{\mathrm{BMO}_{k}}
$$

where the implied constants depend only on $k$ and $\sup _{n} \gamma_{k}\left(\mathscr{F}_{n}\right)$.

Remark 4.3 We close with a few remarks concerning the above result and we assume that $\left(\mathscr{F}_{n}\right)$ is a sequence of increasing interval $\sigma$-algebras with $\sup _{n} \gamma_{k}\left(\mathscr{F}_{n}\right)<\infty$. 


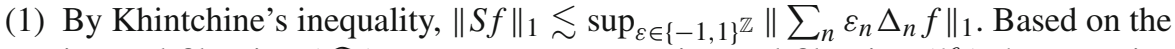
interval filtration $\left(\mathscr{F}_{n}\right)$, we can generate an interval filtration $\left(\mathscr{G}_{n}\right)$ that contains $\left(\mathscr{F}_{n}\right)$ as a subsequence and each $\mathscr{G}_{n+1}$ is generated from $\mathscr{G}_{n}$ by dividing exactly one atom of $\mathscr{G}_{n}$ into two atoms of $\mathscr{G}_{n+1}$. Denoting by $P_{n}^{\mathscr{G}}$ the orthogonal projection operator onto $\mathscr{S}_{k}\left(\mathscr{G}_{n}\right)$ and $\Delta_{j}^{\mathscr{G}}=P_{j}^{\mathscr{G}}-P_{j-1}^{\mathscr{G}}$, we can write

$$
\sum_{n} \varepsilon_{n} \Delta_{n} f=\sum_{n} \varepsilon_{n} \sum_{j=a_{n}}^{a_{n+1}-1} \Delta_{j}^{\mathscr{G}} f
$$

for some sequence $\left(a_{n}\right)$. By using inequalities (2.7) and (2.6) and writing $\left(S^{\mathscr{G}} f\right)^{2}=\sum_{j}\left|\Delta_{j}^{\mathscr{G}} f\right|^{2}$, we obtain for $p>1$

$$
\|S f\|_{1} \lesssim\left\|S^{\mathscr{G}} f\right\|_{1} \leq\left\|S^{\mathscr{G}} f\right\|_{p} \lesssim\|f\|_{p} .
$$

This implies $L_{p} \subset H_{1, k}$ for all $p>1$ and, by duality, $\mathrm{BMO}_{k} \subset L_{p}$ for all $p<\infty$.

(2) If $\left(\mathscr{F}_{n}\right)$ is of the form that each $\mathscr{F}_{n+1}$ is generated from $\mathscr{F}_{n}$ by splitting exactly one atom of $\mathscr{F}_{n}$ into two atoms of $\mathscr{F}_{n+1}$ and under the condition $\sup _{n} \gamma_{k-1}\left(\mathscr{F}_{n}\right)<\infty$ (which is stronger than $\sup _{n} \gamma_{k}\left(\mathscr{F}_{n}\right)<\infty$ ), it is shown in [9] that

$$
\|S f\|_{1} \simeq\|f\|_{H_{1}}
$$

where $H_{1}$ denotes the atomic Hardy space on $[0,1]$, i.e. in this case, $H_{1, k}$ coincides with $H_{1}$.

Acknowledgements Open access funding provided by Austrian Science Fund (FWF). It is a pleasure to thank P. F. X. Müller for very helpful conversations during the preparation of this paper. The author is supported by the Austrian Science Fund (FWF), Project F5513-N26, which is a part of the Special Research Program "Quasi-Monte Carlo Methods: Theory and Applications".

Open Access This article is distributed under the terms of the Creative Commons Attribution 4.0 International License (http://creativecommons.org/licenses/by/4.0/), which permits unrestricted use, distribution, and reproduction in any medium, provided you give appropriate credit to the original author(s) and the source, provide a link to the Creative Commons license, and indicate if changes were made.

\section{References}

1. Asmar, N., Montgomery-Smith, S.: Littlewood-Paley theory on solenoids. Colloq. Math. 65(1), 69-82 (1993)

2. Benedek, A., Panzone, R.: The space $L^{p}$, with mixed norm. Duke Math. J. 28, 301-324 (1961)

3. Bourgain, J.: Embedding $L^{1}$ in $L^{1} / H^{1}$. Trans. Am. Math. Soc. 278(2), 689-702 (1983)

4. Delbaen, F., Schachermayer, W.: An inequality for the predictable projection of an adapted process. In: Séminaire de Probabilités, XXIX, volume 1613 of Lecture Notes in Mathematics, pp. 17-24. Springer, Berlin (1995)

5. De Vore, R.A., Lorentz, G.G.: Constructive approximation, volume 303 Grundlehren der Mathematischen Wissenschaften [Fundamental Principles of Mathematical Sciences]. Springer, Berlin (1993)

6. Diestel, J., Uhl Jr., J.J.: Vector Measures. American Mathematical Society, Providence (1977). (With a foreword by B. J. Pettis, Mathematical Surveys, No. 15) 
7. Fefferman, C.: Characterizations of bounded mean oscillation. Bull. Am. Math. Soc. 77, 587-588 (1971)

8. Garsia, A.M.: Martingale Inequalities: Seminar Notes on Recent Progress. Mathematics Lecture Notes Series. W. A. Benjamin, Inc., Reading (1973)

9. Gevorkyan, G., Kamont, A., Keryan, K., Passenbrunner, M.: Unconditionality of orthogonal spline systems in $H^{1}$. Stud. Math. 226(2), 123-154 (2015)

10. Herz, C.: Bounded mean oscillation and regulated martingales. Trans. Am. Math. Soc. 193, 199-215 (1974)

11. Keryan, K., Passenbrunner, M.: Unconditionality of Periodic Orthonormal Spline Systems in $L^{p}$ (2017). arXiv:1708.09294 (to appear in Studia Mathematica)

12. Lépingle, D.: Une inégalité de martingales. In: Séminaire de Probabilités, XII (University of Strasbourg, Strasbourg, 1976/1977), Volume 649 of Lecture Notes in Mathematics, pp. 134-137. Springer, Berlin (1978)

13. Müller, P.F.X.: A decomposition for Hardy martingales. Indiana Univ. Math. J. 61(5), 1801-1816 (2012)

14. Müller, P.F.X., Passenbrunner, M.: Almost Everywhere Convergence of Spline Sequences (2017). arXiv: 1711.01859

15. Neveu, J.: Discrete-Parameter Martingales, revised edn. North-Holland Publishing Co., Amsterdam (1975). (Translated from the French by T. P. Speed, North-Holland Mathematical Library, Vol. 10)

16. Passenbrunner, M.: Unconditionality of orthogonal spline systems in $L^{p}$. Stud. Math. 222(1), 51-86 (2014)

17. Passenbrunner, M.: Orthogonal projectors onto spaces of periodic splines. J. Complex. 42, 85-93 (2017)

18. Passenbrunner, M.: Spline Characterizations of the Radon-Nikodým Property (2018). arXiv: 1807.01861

19. Passenbrunner, M., Shadrin, A.: On almost everywhere convergence of orthogonal spline projections with arbitrary knots. J. Approx. Theory 180, 77-89 (2014)

20. Pisier, G.: Martingales in Banach Spaces. Volume 155 of Cambridge Studies in Advanced Mathematics. Cambridge University Press, Cambridge (2016)

21. Schumaker, L.L.: Spline Functions: Basic Theory. Pure and Applied Mathematics. Wiley, New York (1981)

22. Shadrin, A.: The $L_{\infty}$-norm of the $L_{2}$-spline projector is bounded independently of the knot sequence: a proof of de Boor's conjecture. Acta Math. 187(1), 59-137 (2001)

23. Stein, E.M.: Singular Integrals and Differentiability Properties of Functions. Princeton Mathematical Series, No. 30. Princeton University Press, Princeton (1970)

24. Stein, E.M.: Topics in harmonic analysis related to the Littlewood-Paley theory. Annals of Mathematics Studies, No. 63. Princeton University Press, Princeton (1970)

25. von Golitschek, M.: On the $L_{\infty}$-norm of the orthogonal projector onto splines. A short proof of A. Shadrin's theorem. J. Approx. Theory 181, 30-42 (2014)

Publisher's Note Springer Nature remains neutral with regard to jurisdictional claims in published maps and institutional affiliations. 